\title{
Towards Services-Based Enterprise Architecture for Cloud Computing-Opened Information Systems
}

\author{
M'barek El Haloui*, Houda Kriouile, Abdelaziz Kriouile \\ IMS Team, SIME Laboratory, ENSIAS, Mohammed V University, Rabat, Morocco, Moroccan. \\ * Corresponding author. Tel.: +212 661131070; email: mbarek.haloui@um5s.net.ma \\ Manuscript submitted March 5, 2015; accepted May 11, 2015. \\ doi: $10.17706 /$ jcp.10.3.195-202
}

\begin{abstract}
With the development of cloud computing technologies and the multiplication of enterprise architecture reference frameworks, the industrials are encouraged to take into account the new concepts of cloud service in the evolution of their information system strategies. The present paper starts by presenting background concepts and the state of the art of the enterprise architecture, in order to propose a maturity model that helps enterprises to build and evaluate a functional component of their Information System architecture and allow them to qualify its externalization to the Cloud Computing as well as its monitoring.
\end{abstract}

Key words: Cloud computing, enterprise architecture, information systems, IS reference framework, IT service management.

\section{Introduction}

Due to the rapid changing of information technologies (IT), the continuous transformation of IT systems, and particularly the emergence of cloud computing, the industrials are encouraged to evolve their information systems (IS) continuously and rapidly to take advantage of offered services. The alignment of these systems to the strategic choices of the enterprise remains a major concern and involves a permanent modification in the perimeter of the IS architecture, which requires continuous adaptation of its initial partition. The IS urbanization or the enterprise architecture (EA) approaches allow to define the evolution plan of the enterprise's IS as well as its governance in the medium and long term. However, the choice of partial or total opening of the information system to cloud computing requires having an anticipative vision of IS evolution as well as a maturity level of IT architecture's components and monitoring processes of services provided to the end user.

The remainder of this paper is structured as follows. Section 2 introduces the information system definition as well as its alignment with the enterprise strategy. Section 3 exposes an overview of EA concepts and models. Section 4 describes the cloud computing and its relation with the EA. Section 5 provides a description and example of architecture model for IT service management. Section 6 introduces our contribution by the proposition of a maturity-based transformation model for enterprise architecture functional components. Finally, Section 7 is a conclusion of the paper.

\section{Information System and Its Alignment with the Enterprise Strategy}

\subsection{Information System}

The review of domain's literature reports that there are several definitions of the IS, depending on 
researcher viewpoints, experiences, and contexts. Indeed, defining the IS as a Work System is considered to be the most appropriate definition [1]. This definition allows identifying the components of an IS and eliminates any confusion with a computer system [2]. S. Alter defines the Work System as "a system in which human participants and/or machines perform work (processes and activities) using information, technology, and other resources to produce specific products and/or services for specific internal and/or external customers." [1]. Fig. 1 illustrates the components of a work system. The IS is defined as a work system whose internal functions are limited to treating the information by running six types of operations, which are capture, transmit, store, retrieve, manipulate, and display the information. An IS produces information, assists, or automates the work performed by other work systems [3]. In other words, an IS is a work system whose processes and activities are devoted to the information treatment, namely the capture, the transmission, the storage, the retrieval, the manipulation, and the display of information [3].

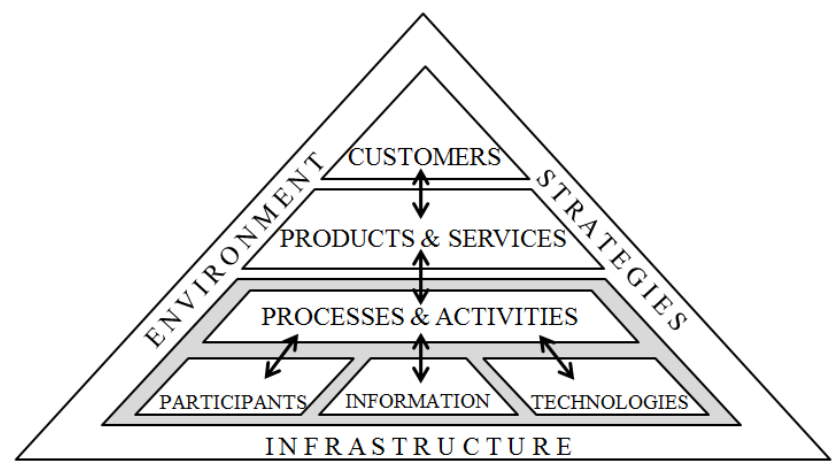

Fig. 1. The work system framework [3].

\subsection{Information System and the Enterprise Strategy}

J. Simonin considers that the enterprise sustainability is valorized by the integration of its strategy in the enterprise's processes that are used to produce value. He adds that a sustainable Information System is valorized by the integration of this strategy in enterprise IS to produce value [4]. The strategy includes two aspects: the work system strategy and the enterprise strategy [3]. The strategies that are relevant to a work system include the enterprise strategy, the organizational strategy, and the work system strategy. In general, the three levels strategies must be aligned and the work system strategy must support the remaining strategies [1]. The strategy is modeled by a hierarchy of objectives. The latter are decomposed into sub-objectives until all objectives can be achieved by at least one process [5].

\section{Enterprise Architecture or Information System Urbanism}

\subsection{Enterprise Architecture vs. Information System Urbanization}

The Enterprise Architecture (EA) is a base of strategic information asset, which defines the mission, the information needed to carry out the mission, the technology required to perform the mission, and the transition process of the implementation of new technologies in response to the evolving of the mission [6], [7].

The information system urbanization (ISU) is a concept inspired by the town planning and used in information systems. The ISU concept was developed in France by enterprises of significant size in the early 90s [4], [5]. It has started with Jacque Sassoon's publication of the book "Information Systems Urbanism" in 1988 [5]. This concept has then evolved over the years especially with its promotion by specialized communities such as CIGREF and Club-Urba. The EA is a framework of an Anglo-Saxon origin which took start from John Zachman publication of the paper "A Framework for Information System" in IBM System 
Journal in 1987 [5].

Several EA frameworks and methods are available, e.g.: Zachman, E2AF, AEP, FAEF, TAEF, TOGAF, IAF, JTA, DodAF, TAFIM, CIMOSA, PERA, SAGA, etc. Most of which are derived from Zachman's Framework [8]. A French EA approach, entitled ISU ("Urbanisation des Systèmes d'Information") [5], [8] has also been added. Existing approaches still suffer from some issues [8] and this because of the fact that they represent frameworks of reference. These limits are summarized as: lack of integration, lack of formal definition, lack of documentation of the EA management process, and no support for improving the strategic alignment.

A study by the IFAES (Institute for EA Development) [8], [9] shows that two main reasons to undertake the enterprise architecture are: the business IT alignment and the construction of a set of transformation instructions.

The ISU approach allows to make IS more able to serve the enterprise's strategy and anticipate changes in its environment [10]. This is the case of a meta-model allowing describing all the knowledge of an IS according to five IS views [11] (Fig. 2):

- A strategy view, describing the strategic missions and objectives of the IS;

- A business view, describing the processes, activities, and organization penetrated;

- A functional view, describing the IS use cases and the information manipulated independently of technological choices;

- An applicative view, describing software components (e.g., applications) kept as well as their interdependencies (flux, software architecture, etc.);

- An infrastructure view, describing the physical resources and the deployment.

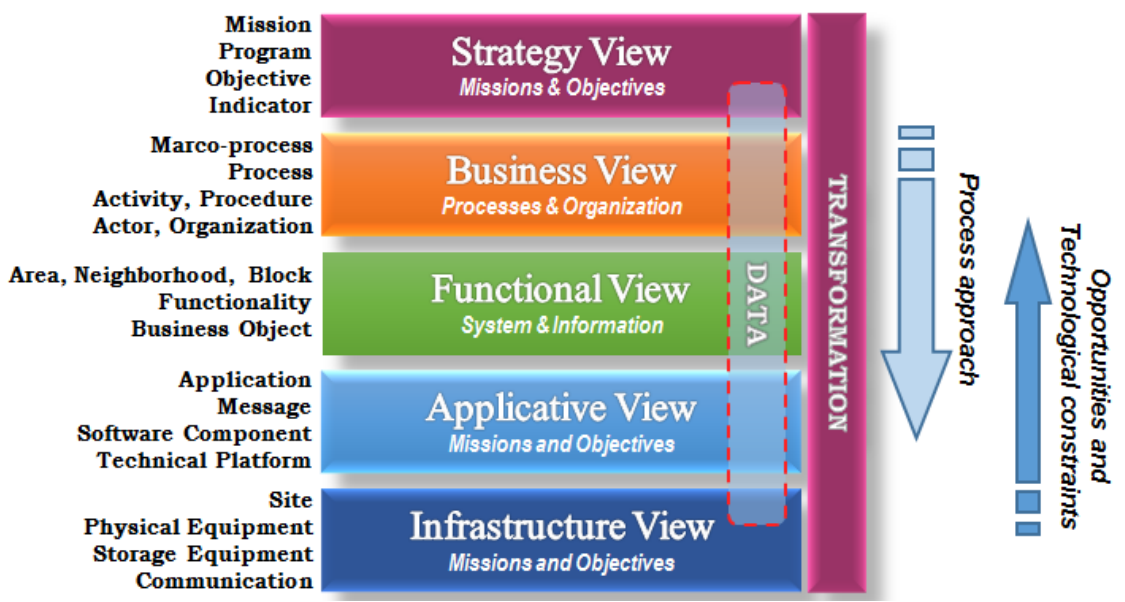

Fig. 2. An information system architecture [5], [11].

This partition of the IS in points of view allows to structure the urbanization process. Also, it allows putting a common language, which is an essential prerequisite for the establishment of a common framework for discussion, cooperation, and decision. The number of layers and their communication is not yet a standard. Although a strong evolution of the maturity of the proposed solutions and a convergence of practices is clearly observed.

\subsection{Urbanization Approach}

The ISU approach consists in transforming, pooling, reusing and aligning the assets (and resources) of an organization (equipment, personnel, projects, processes, data) with its own operational characteristics, its scalability strategy and its stress field, all in a formal, understandable and shared framework [11]. The urbanization approach adopted by the Club-Urba is structured around several activities organized under such a framework which is illustrated in Fig. 3 [11]. It is articulated around three kinds of activities: the 
piloting activities, core business activities, and support activities.

From another point of view, the most practiced approach, The Open Group Architecture Framework (TOGAF), has been proposed in 1995 by the Open Group enabling users to implement open solutions at lower costs, to simplify design, planning, acquisition, and open systems integration processes, and to help IS directors to better communicate their goals and strategies to the decision makers [8]. This approach is based on a EA building method called Architecture Deployment Method (ADM).

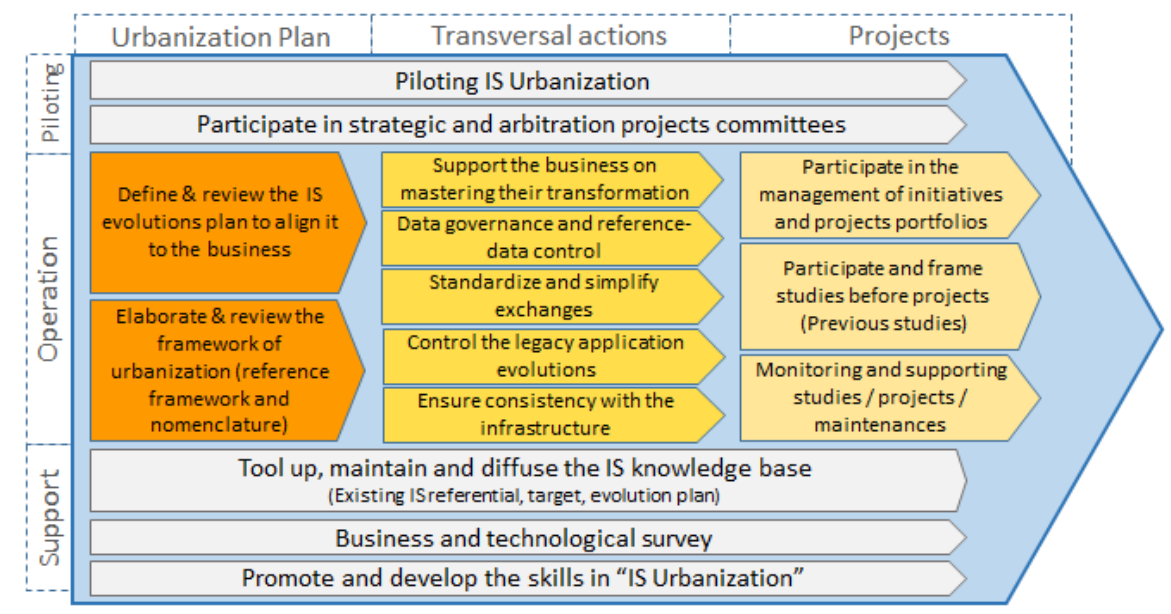

Fig. 3. Club-Urba urbanization approach [11].

Fig. 4 shows the ADM phases. Among the feedbacks on the TOGAF approach [8], the central position of requirements constitutes a thread that ensures the continuity between all models and phases of the methodological process.

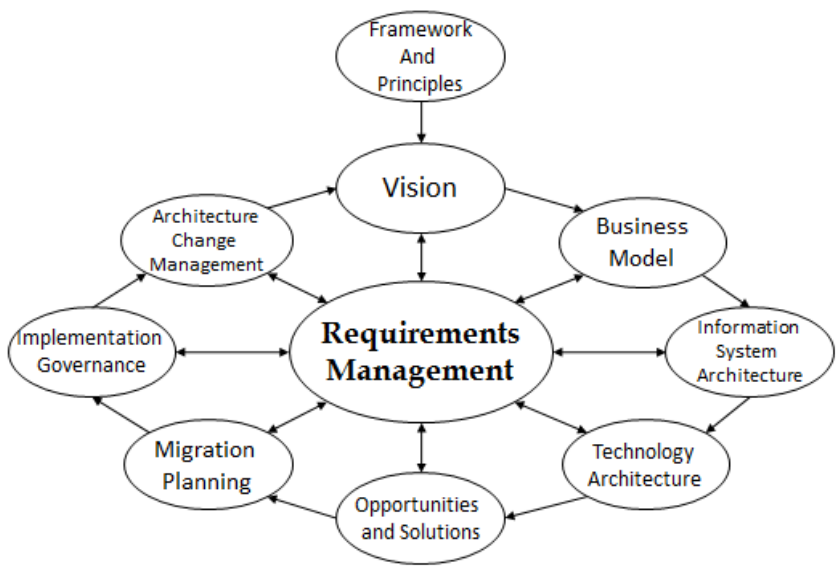

Fig. 4. The ADM of TOGAF approach.

\section{The Cloud Computing and Enterprise Architecture}

\subsection{Cloud Definition}

Through a multiple definitions study, [12] defines the cloud computing as "a large pool of easily usable and accessible virtualized resources (such as hardware, development platforms and/or services). These resources can be dynamically reconfigured to adjust to a variable load (scale), allowing also for an optimum resource utilization. This pool of resources is typically exploited by a pay-per-use model in which guarantees are offered by the Infrastructure Provider by means of customized Service Level Agreements (SLA)."

The National Institute of Standards and Technology (NIST) defines the Cloud Computing as "a model for 
enabling ubiquitous, convenient, on-demand network access to a shared pool of configurable computing resources (e.g., networks, servers, storage, applications, and services) that can be rapidly provisioned and released with minimal management effort or service provider interaction" [13]. [14] consider this definition the most adopted. The NIST defines three service models for the Cloud Computing [13]:

- Software as a Service (SaaS): The capability provided to the consumer is to use the provider's applications running on a cloud infrastructure.

- Platform as a Service (PaaS): The capability provided to the consumer is to deploy onto the cloud infrastructure consumer-created or acquired applications created using programming languages, libraries, services, and tools supported by the provider.

- Infrastructure as a Service (IaaS): The capability provided to the consumer is to provision processing, storage, networks, and other fundamental computing resources where the consumer is able to deploy and run arbitrary software, which can include operating systems and applications.

Currently, a new concept of cloud service models is trendy [14]-[16] which is the anything or everything as a Service (XaaS), such as Storage as a Service (StaaS), Testing as a Service (TaaS), Hardware as a Service (HaaS), Framework as a Service (FaaS), etc. NIST identifies four Cloud Computing deployment models [13]: private cloud, community cloud, public cloud, and hybrid cloud.

\subsection{Domains-Based Enterprise Architecture: A Functional View}

The functional IS segmentation [5], inspired by the concept of towns planning, is based on a five major domains model (Fig. 5):

- The "Operation" domain includes all functions and business objects that have a guidance, a purpose, to serve users (external actors).

- The "Piloting \& Control" domain includes all the functions and cross-piloting business objects of the enterprise's activities, as well as control functions (audit, inspection ...).

- The "Resource \& Support" domain includes all functions and business objects of down force or support to other domains. These are mainly resource management functions: HR, Finance, Real Estate, Generals means, IT ...

- The "Exchange" domain includes all the functionalities and business objects relating to trade between the various contributors actors, users, partners, clients of the enterprise IS.

- The "Transversal Data" domain groups and isolates all data -and functions that manipulate it- which are transverse or common to most, if not all, areas of the other domains.

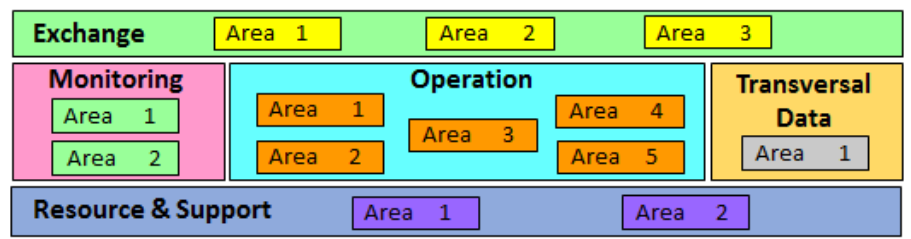

Fig. 5. IS functional architecture [5], [11].

\subsection{Enterprise Architecture and Cloud Computing}

From the perspective of standardization and interoperability, several cloud reference architecture model were developed as IBM Cloud Computing Reference Architecture [16]. Though, a standard architecture still lacks [15]. However, current models focus on the cloud provider and generally do not adequately address the enterprise side and its characteristics [14], [17]. Prasad et al. specify requirements of cloud architecture choice from enterprise, end-user, and service provider sides [15]. The identified requirements are as follows: service delivery model (SaaS, PaaS, IaaS), service-centric, data management and storage, 
virtualization management, cloud deployment, fault tolerance, security, quality of service, cloudonomics, load balancing, interoperability, scalability, data governance, data migration, business process management, third party engagement, transferable skills, user consumption-based billing and metering, user-centric privacy, service level agreements, adaptability and learning, and user experience. Other requirements and best practices are also recommended for migrating to cloud environments such as: application security and data encryption [6]. A cloud-opened EA should take account of these requirements to allow flexible migration of its functional blocks towards and cross cloud service providers platforms.

\section{Services Management}

Reference [17] proposes a service management architecture composed of the traditional IT management processes as defined by standards such as IT Infrastructure Library (ITIL), a service life-cycle management layer, and an infrastructure resources management layer (Ensembles).

The cloud service life-cycle is composed of the following steps (Fig. 6):

- Definition: all management knowledge required for a specific type of cloud service is captured in a service template. A model service template contains all the knowledge that is needed to instantiate a cloud service and manage the outcome cloud service instances.

- Offering: a cloud service offering is created based on a service template by adding all information specific to the cloud service provider and offering.

- Subscription and instantiation: From a service catalog, a requester can select an offering and then specify the necessary settings for the service instance, such as capacity, availability, performance and duration.

- Production: The phase in which the service instance is managed either automatically based on defined SLA or manually by an administrator from the service provider side or the consumer side.

- Termination: When a Cloud service instance is no longer needed or when the reservation for its resources expires, it is terminated either automatically or manually by an administrator.

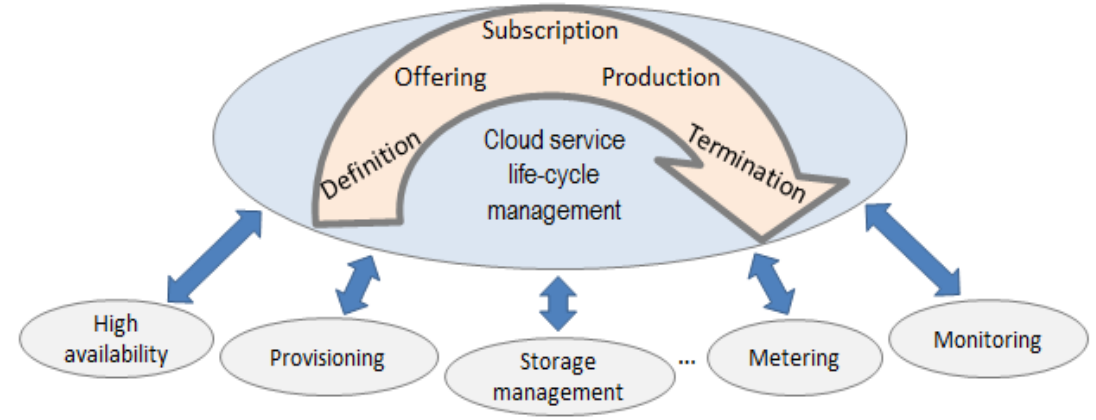

Fig. 6. Cloud services management layers [17].

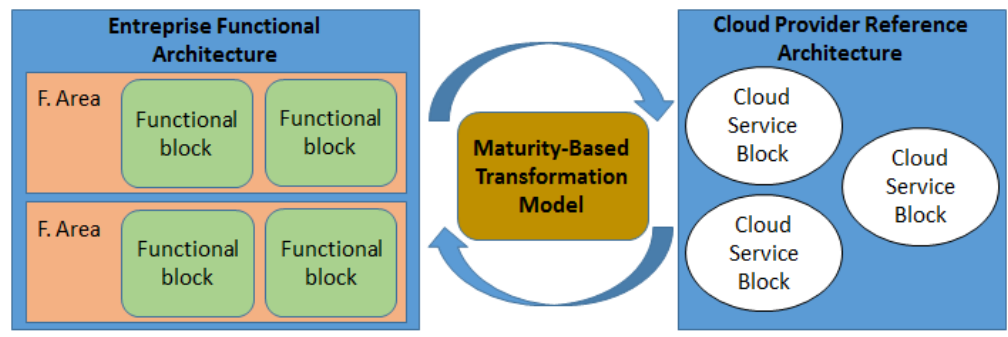

Fig. 7. Maturity-based transformation model.

\section{Discussion}


Among the research work in the domain of EA-based cloud services, some works raised the need of evaluation and decision criteria for the adoption of cloud computing within the enterprises [18]. The majority of current approaches focus on the EA of the service provider [14]. Therefore, there is a need to give the companies a way to assess the components of its IS and to measure its functional maturity, then decide to migrate or not a component to the cloud and to choose the adequate kind of cloud. Fig. 7 provides an overview of our proposed model that provides a mean which enables to design a mature and cloud-opened IS. The proposed model constitutes a decision support tool for IT evolution strategy of the enterprise.

\section{Conclusion}

Subscription to the cloud offers has now become a competitive alternative in enterprise information systems. The enterprises need a fast and flexible assessment tools to take advantages of these services. That is why the proposal of such transformation models is an added value that reduces the gap between the rapid advancement of IT technologies and the IS agility.

\section{References}

[1] Alter, S. (2008). Defining information systems as work systems: Implications for the IS field. European Journal of Information Systems, 17, 448-469.

[2] Maallam, M., \& Kriouile, A. (2012). A model of maturity for IS risk management case study. Computer and Information Science, 5(3).

[3] Alter, S. (2002). The work system method for understanding information systems and information system research. Communications of the Association for Information Systems, 9, 90-104.

[4] Simonin, J. (2009). Architecture design of a System Drived by a Functional Urbanism Model. Thesis no. 3844, University of Rennes 1.

[5] Longépé, C. (2009). The Enterprise Architecture IT Project: The Urbanisation Paradigm (4th ed.). Dunod.

[6] Aureli, L., Pierfranceschi, A., \& Wache, H. (2012). Enterprise architectures for cloud computing. Proceedings of the IEEE Fifth International Conference on Cloud Computing.

[7] Linthicum, D. (2010). Relevance of Enterprise Architecture to Cloud Computing. From http://www.ebizq.net/blogs/cloudsoa/2010/12/relevance-of-enterprise-architecture-to-cloud-comp uting.php

[8] Salensi, C., et al. Enterprise Architecture, Practical Problems to Innovation. From http://www.researchgate.net/...Enterprise_architecture.../09e415086e10b9007e

[9] Schekkerman, J. (2005). Trends in Enterprise Architecture 2005 - How are Organizations Progressing? Institute for Enterprise Architecture Development (IFEAD) Report.

[10] CIGREF. (2003). Accroître L'agilité du systeme D'information, Livre blanc. From http://www.cigref.fr/cigref_publications/RapportsContainer/Parus2003/2003_-_Accroitre_l_agilite_d u_systeme_d_information_web.pdf

[11] Common framework of IS urbanism of the State-France. From https://references.modernisation.gouv.fr/.../Cadre\%20Commun\%20d'Urbanisation

[12] Vaquero, L. M., Rodero-Merino, L., Caceres, J., \& Lindner M. (2008). A break in the clouds: Towards a cloud definition. From http://ccr.sigcomm.org/online/.../p50-v39n1l-vaqueroA.pdf

[13] NIST. (2011). Computing Standards Road Map. NIST publication.

[14] El Houssaini, C., Kriouile, A., \& Nassar, M. (2014). A Governance Based Architecture for Enterprise Cloud Computing Adoption. International Journal of Cloud Applications and Computing, 4(3), 54-67.

[15] Prasad, R. B., Jukan, A., Katsaros, D., \& Goeleven, Y. (2011). Architectural requirements for cloud 
computing systems: An enterprise cloud approach. Journal of Grid Computing, 9, 3-26.

[16] IBM cloud computing reference architecture.

From https://www.ibm.com/developerworks/community

[17] Breiter, G., \& Behrendt, M. (2009). Life cycle and characteristics of services in the world of cloud computing. IBM Journal of Research and Development, 53(4), 3.

[18] Wang, H., He, W., \& Wang, F.-K. (2012). Entreprise cloud service architecture. Proceedings of IEEE 3rd International Conference on Cloud Computing (pp. 27-34).

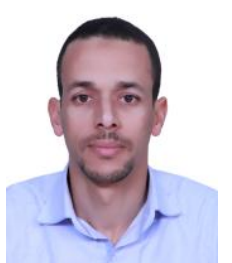

M'barek El Haloui is a Ph.D. candidate, and a member of the IMS team from the SIME laboratory, National School of Computer Science and Systems Analysis (ENSIAS), Mohammed V University of Rabat, Morocco. He received the engineer degree in computer science and network systems engineering in 2004 from the Mohammadia School of engineering (EMI) at Mohammed V University of Rabat, Morocco.

He excises the job of consulting and audit of information systems from 2005 to 2009 the. Actually, he is a project manager at a telco. company and manage currently a cloud computing project.

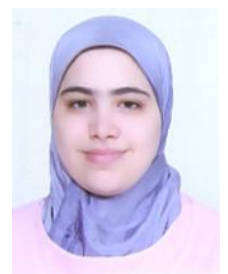

Houda Kriouile was born at Nancy in France, in 1990. She is a Ph.D. candidate, and a member of the IMS team from the SIME laboratory, National Higher School for Computer Science and Systems Analysis (ENSIAS), Mohammed V University of Rabat, Morocco. She received her engineer degree in computer science and software engineering in 2012 from the ENSIAS School at Mohammed V University of Rabat, Morocco.

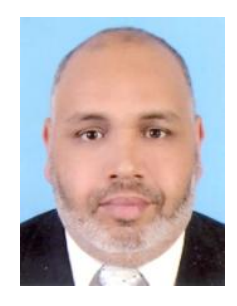

Abdelaziz Kriouile was born at KMB city, in 1961. He is a full professor in the Software Engineering Department, National Higher School for Computer Science and Systems Analysis (ENSIAS), Rabat, Morocco. He is the head of SIME laboratory. He received his Ph.D. degree in computer Science from the Nancy University, France. His research activities focus on information systems, cloud computing, and context-aware service-oriented computing. He leads numerous projects related to the application of these domains. 